\title{
Eclipse Timing Variations of Planets in P-Type Binary Star Systems
}

\author{
Richard Schwarz ${ }^{1}$, Nader Haghighipour ${ }^{2}$, Barbara Funk ${ }^{1}$, \\ Siegfried Eggl ${ }^{1}$, and Elke Pilat-Lohinger ${ }^{1}$ \\ ${ }^{1}$ Institute for Astronomy, University of Vienna, \\ Türkenschanzstrasse 17, 1180 Austria \\ email: schwarz@astro.univie.ac.at \\ ${ }^{2}$ Institute for Astronomy and NASA Astrobiology Institute, University of Hawaii \\ 2680 Woodlawn Dr., Honolulu, HI, 96822 USA
}

\begin{abstract}
In close eclipsing binaries, measurements of the variations in the binary's eclipse timing may be used to infer information about the existence of planets in P-Type motion. To study the possibility of detecting such planets with CoRoT and Kepler, we calculated eclipse timing variations (ETV) for different values of the mass and orbital elements of the perturbing planet. These investigations are a continuation of the work of Schwarz et al. (2011).
\end{abstract}

Keywords. methods: numerical, binaries: eclipsing, planetary systems, planets: detection

\section{Introduction}

Today (June 2011), we know 53 extrasolar planets in 39 binary systems and 7 extrasolar planets in 7 triple star systems. In general, one may distinguish different types of stable orbits for planets in binary systems (Dvorak 1988):

- S-Type: The planet moves around one of the two stars.

- P-Type: The planet moves around the entire binary.

- L-Type: The planet moves close to the equilibrium points $\left(L_{4}\right.$ and $\left.L_{5}\right)$ of a binary like Jupiter's Trojans. Possible candidates were presented in the work of Schwarz et al. (2009).

In the study by Schwarz et al. (2011), the dynamics of a binary star system with a close P-Type planet, has been analysed and the eclipse timing variations (ETV) were calculated for different values of the mass ratio and orbital elements of the binary system. In this study, we investigated P-Type planets farther away from the binary (up to $5 \mathrm{AU}$ ).

\section{Model and methods}

Our numerical simulations were carried out using the Lie-Series-method. As dynamical model, we used the full three-body problem and considered the binary system in circular motion with an initial separation of $0.05 \mathrm{AU}$ and a mass of $m_{1}=m_{2}=1 M_{\text {sun }}\left(m_{1}\right.$ is the mass of the primary star and $m_{2}$ the mass of the secondary star). The planet moves in a far away circular orbit around both stars and has masses of 1 and 10 Jupiter-masses $\left(M_{J}\right)$. Gravitational interactions between the binary and the planet will perturb the star's motion, resulting in a deviation from their Keplerian orbits. In an eclipsing binary, these deviations result in variations in the time and duration of the eclipse. We compared the expected amplitude of the $\operatorname{ETV}\left(\sigma=\left(d_{t \max }-d_{t \min }\right) / 2\right)$ with the detection limit of current space observatories. With the amplitude $\sigma=16 \mathrm{sec}$ for CoRoT (at $\mathrm{L}=15.5$ 
mag) and for Kepler $\sigma=4 \sec ($ at $\mathrm{L}=14.5 \mathrm{mag}$ ), we may show if the ETV signals of our calculations are detectable or not.

\section{Results}

If the ETV signal is higher than the observational threshold, we can assume that a planet will be indirectly detectable by the eclipse timing measurements of the secondary star (Schwarz et al. 2011). There are 2 dynamical effects which change the ETV signal, caused by the perturbation of an additional planet:

(a) Short term variations in the binary's period. Former investigations showed (Schwarz et al. 2011) that the planets have to be in circular orbits very close to the secondary star $a=0.1 \mathrm{AU}$ or, in the case of planets with a larger distance $a>0.1 \mathrm{AU}$, they have to be more massive $\left(m>5 M_{J}\right)$ to produce detectable ETV signals.

(b) The binary stars also perform an orbit around the common barycenter, leading to different light travel times.

The results of our investigations are presented in Figure 1 for a mass of the planet of $1 M_{J}$ (left graph) and $10 M_{J}$ (right graph). We compared the amplitude $(\sigma)$ of the ETV signal for the two different effects as follows: the solid line marks the signal with the first effect and the dashed line represents the second effect. Thus, we can conclude that planets which are very close $(a \leqslant 0.3 \mathrm{AU})$ to the binary system are dominated by the first effect (Schwarz et al. 2011), whereas planets which are far $(a>0.3 \mathrm{AU})$ from the binary are dominated by the second effect, this is true for both masses of the planet.
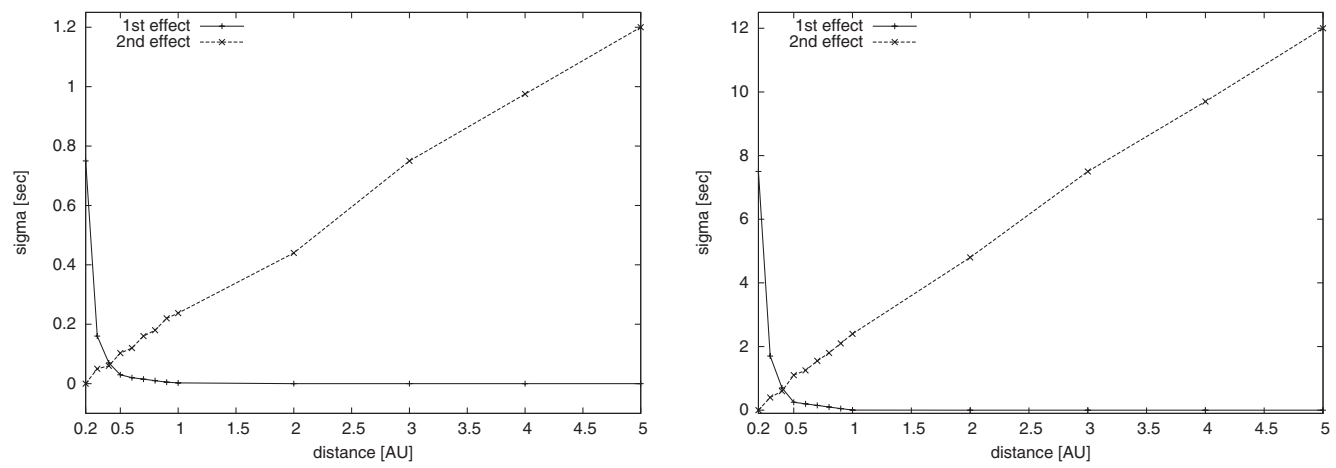

Figure 1. Amplitude $(\sigma)$ of the ETV signals for planets with $1 M_{J}$ (left graph) and 10 $M_{J}$ (right graph) for different distances to the binary.

\section{Acknowledgement}

Support was received from the ÖFG projects 06/12290 for R.S. and 06/12277 for B.F., the NASA Astrobiology Institute for N.H., the Austrian FWF project no. P20216 for S.E. and the Austrian FWF projects no. P20216 and P22603 for E. P-L.

\section{References}

Dvorak, R., 1984, CeMDA, 34, 369

Schwarz, R., Süli, Á., \& Dvorak, R. 2009, MNRAS, 398, 2085

Schwarz, R., Haghighipour, N., Eggl, S., Pilat-Lohinger, E., \& Funk, B., 2011, MNRAS, 414, 2763. 